\title{
Photodynamic Therapy For Synovial Hyperplasia in Patients With Refractory Rheumatoid Arthritis : A Study Protocol For A Randomised Double-Blind Blank-Controlled Prospective Trial
}

\section{Xiaofeng Zhao}

Integrated Hospital of Traditional Chinese Medicine,Southern Medical University

\section{Ensheng CHEN}

Integrated Hospital of Traditional Chinese Medicine,Southern Medical University

Fangfang ZUO

Integrated Hospital of Traditional Chinese Medicine, Southern Medical University

Yanan BI

Integrated Hospital of Traditional Chinese Medicine,Southern Medical University

\section{Yanyan CAO}

Integrated Hospital of Traditional Chinese Medicine,Southern Medical University

\section{Yi YUAN}

Integrated Hospital of Traditional Chinese Medicine,Southern Medical University

\section{Kaiqin LI}

Integrated Hospital of Traditional Chinese Medicine,Southern Medical University

\section{Yanan XUAN}

Integrated Hospital of Traditional Chinese Medicine,Southern Medical University

Libo LI

Integrated Hospital of Traditional Chinese Medicine,Southern Medical University

\section{Lijuan WAN}

Integrated Hospital of Traditional Chinese Medicine,Southern Medical University

\section{Xiangqun ZHANG}

Integrated Hospital of Traditional Chinese

\section{Feifei YAN}

Integrated Hospital of Traditional Chinese Medicine,Southern Medical University Jiyuan ZHOU

School of Public Health,Southern Medical University

\section{Kun YIN}

General OUTpatient Clinic of Chigang Street Community Health Service Centre

Changhong XIAO ( $\nabla$ nfzxyfsbk@163.com) 
Integrated Hospital of Traditional Chinese Medicine,Southern Medical University https://orcid.org/0000-0003-2715-1960

\section{Research Article}

Keywords: refractory rheumatoid arthritis (RRA), photodynamic therapy (PDT), UCSS, Persistent synovial hyperplasia

Posted Date: April 12th, 2021

DOl: https://doi.org/10.21203/rs.3.rs-340779/v1

License: (9) This work is licensed under a Creative Commons Attribution 4.0 International License. Read Full License 


\title{
Photodynamic therapy for synovial hyperplasia in patients
} with refractory rheumatoid arthritis: a study protocol for a

\section{randomised double-blind blank-controlled prospective trial}

Xiaofeng $Z H A O^{1 \triangle}$, Ensheng $C H E N^{1 *}$, Fangfang $Z U O^{1}$, Yanan $\mathrm{BI}^{1}$, Yanyan $C A \mathrm{O}^{1}, Y i Y_{\mathrm{Y}} \mathrm{CN}^{1}$, Kaiqin $\mathrm{LI}^{1}$, Yanan XUAN ${ }^{1}$, Libo $\mathrm{LI}^{1}$, Lijuan WAN ${ }^{1}$, Xiangqun ZHANG ${ }^{1}$, Feifei $Y A N^{1}$, Jiyuan $Z H O U^{2}$, Kun Yin ${ }^{3}$, Changhong XIAO ${ }^{1 \#}$

1. Integrated Hospital of Traditional Chinese Medicine, Southern Medical University, Guangzhou, China.

2. Department of Biostatistics, School of Public Health, Southern Medical University, Guangzhou, China.

3. General Outpatient Clinic of Chigang Street Community Health Service Centre, Guangzhou, China.

\#Corresponding author: Changhong Xiao, Professor, chief physician, doctoral supervisor, Tel: 86-020-61650916, e-mail: nfzxyfsbk@163.com.

*Co-first author: Ensheng Chen, Deputy director of physician, e-mail: 562325605@qq.com. ${ }^{\triangle}$ The first author: Xiaofeng Zhao, Professor, Master's degree, mainly engaged in clinical research of rheumatism,Tel: 86-020-61650914, e-mail: zxf1988yk@163.com.

\begin{abstract}
Background Persistent synovial hyperplasia with inflammation in rheumatoid arthritis is the main cause of refractory rheumatoid arthritis (RRA). As a means of local treatment, photodynamic therapy (PDT) confers less trauma, stronger targeting, and more durable curative effects than steroid injections or arthroscopic synovectomy. The aim of this trial will be to evaluate the short-, medium- and long-term clinical efficacy of PDT in the treatment of RRA synovial hyperplasia and synovitis.
\end{abstract}

Methods and analysis This is a single-centre, randomised, double-blind, blank-controlled, prospective trial. A sample of 126 RRA patients will be randomly divided into 3 groups: the control group, the PDT once group, and the PDT twice group, 42 per group. The trial will be conducted at the Rheumatology and Immunology Department of Integrated Hospital of Traditional Chinese Medicine, Southern Medical University. Assessments at baseline, the first operation, the second operation (4th week), and then at three follow-ups (8th week, 24th week, 48th week) will be performed. The Ultrasound Compound Score of Synovitis (UCSS), knee joint clinical assessments, Disease Activity Score in 28 Joints (DAS28), serological inflammation indexes and specific antibody levels, pathological biopsies of synovial tissue and X-ray assessments of bone destruction will be evaluated. An improvement in the UCSS will be the main endpoint, and the UCSS at the 8th week versus the baseline value will reflect the short-term outcome of the operation. The results of 
the 24th week and 48th week follow-up will reflect the medium- and long-term curative effects, respectively.

Ethics and dissemination The protocol was approved by the Medical Ethics Committee of Integrated Hospital of Traditional Chinese Medicine, Southern Medical University, China (Approval No. of the ethics committee: NFZXYEC-2017-005) and later registered in the Chinese Clinical Trials Registry with Registration number ChiCTR1800014918 (approval date: February 21, 2018). All procedures will be in accordance with Chinese laws and regulations, as well as the WMA Declaration of Helsinki. Any modifications to the protocol will be approved by the Ethics Committee of our hospital.

\section{Trial registration number ChiCTR1800014918.}

\section{Background}

Rheumatoid arthritis (RA) is a chronic inflammatory disease characterised by persistent joint inflammation and synovial hyperplasia. Without adequate treatment, patients with RA will develop joint deformities and progressive functional impairment. With the implementation of treat-to-target strategies and the availability of biologic therapies, the outcomes for patients with RA have significantly improved [1]. However, some patients with RA have a poor response to multiple drugs (the tolerance incidence of first-line drugs is more than $20 \%$, and the effectiveness of various biological agents is only 70\%) [2]. Although most systemic inflammation and arthritis can be controlled, recurrent seizures or persistent synovial hyperplasia of some joints (eg, the elbow, knee, and ankle) lead to bone destruction, which worsens into refractory RA (RRA) a more harmful and difficult to treat condition. Apart from traditional medicine for systemic treatment in the past, local injection with hormones in the joint cavity and arthroscopic synovectomy can also be used to treat synovial hyperplasia. Local intra-articular hormone injection has only a short-term treatment effect but obvious deleterious side effects. The continuous curative effect of long-term treatments is poor and can easily cause inflammation and crystallisation; furthermore, patient compliance is also poor [3]. Arthroscopic synovectomy shows some improvement over steroid injection, but it is a limited invasive treatment that can result in large injuries and cannot completely excise the synovium; additionally, it is unable to change the characteristics of the synovial pathology, and symptoms can recur in half a year or even sooner [4]. There remains a 
need for therapy with long-term efficacy for joint local treatment in the clinic.

Photodynamic therapy (PDT) is a new therapeutic method that can activate a photosensitizer (PS) with a specific wavelength, generate photochemical reactions and produce toxic side effects on surrounding biological tissue [5]. In short, based on the affinities of different photosensitive drugs to different target tissues, photoactivated PSs at specific wavelengths will produce reactive oxygen species (Ros) with cytotoxic effects that can selectively kill rapidly proliferating inflammatory cells. The advantage of this procedure is that it can selectively kill inflammatory cells or tissues but has little or no effect on normal tissues [6].

The goal of applying PDT in the treatment of RRA is to change its characteristic pathology-synoviocyte proliferation and inflammatory cell infiltration-with PS -specific retention in synoviocytes and inflammatory cells, selectively killing the diseased tissues without damaging the normal tissues. PDT has a unique goal and remarkable effects and causes little damage to normal tissue, which has advantages and development prospects that other therapies do not.

Compared with systemic therapy with traditional medicine, PDT treatment for refractory joints, such as the elbow, knee and ankle, can address the poor effects of prior traditional therapies on RRA and improve the clinical curative effects, which would be of great clinical significance to improve the quality of life of RA patients.

\section{Methods}

\subsection{Trial design}

This is a unicentric, randomised, double-blind, sham-operation-controlled trial with 3 groups: a control group, a PDT once group, and a PDT twice group. The curative effect will be compared statistically across groups. In total, there will be 126 patients (42 in each group). The trial will be conducted at the Rheumatology and Immunology Department of Integrated Hospital of Traditional Chinese Medicine, Southern Medical University. Further details can be seen in the flowchart of the trial design in Fig. 1.

The study has been carried out following the Standard Protocol Items: A SPIRIT Checklist has been included.

\subsection{Patients}

2.2.1 RRA diagnostic criteria: (1) The 1987 American Rheumatism Association (ARA) 
classification criteria for RA: i. morning stiffness in and around joints lasting at least 1 hour before maximal improvement; ii. soft tissue swelling (arthritis) of 3 or more joint areas observed by a physician; iii. swelling (arthritis) of the proximal interphalangeal, metacarpophalangeal, or wrist joints; iv. symmetric swelling (arthritis); v. rheumatoid nodules; vi. the presence of rheumatoid factor; and vii. radiographic erosions and/or periarticular osteopenia in hand and/or wrist joints. Criteria $\mathrm{i}$ through iv must have been present for at least 6 weeks. Rheumatoid arthritis is defined by the presence of 4 or more criteria [7]. (2) RA still not responding with low activity (DAS28 $<3.2$ ) or remission (DAS28 $<2.6$ ) $[8,9]$ following regular, sufficient treatment (traditional drugs including non-steroidal anti-inflammatory drugs, more than two DMARDs and/or biological DMARDs, combined therapy for more than 3 months).

2.2.2 The inclusion and exclusion criteria of the patients are presented in Table 1.

Table 1. Inclusion and exclusion criteria

Inclusion criteria

1. Male or female pts (aged between 18 and 70 years)

2. Patients who meet the diagnostic criteria for RRA

3. Patients with at least one side knee swelling for one month

4. Patients with synovial hyperplasia confirmed by ultrasound or MRI: UCSS more than 2 points and/or single-site MR scores more than 2 points

5. Patients capable of signing the informed consent and able to complete the experiment as required

6. Patients with good compliance and who will complete follow-up

Exclusion criteria

1. Patients with serious primary diseases such as those affecting the cardiovascular, liver, kidney, brain or haematopoietic system

2. Patients with infection and abnormal coagulation function

3. Breast-feeding and pregnant women

4. Patients allergic to anaesthetics and photosensitive drugs

5. Patients injected with intra-articular drug in one month

6. Patients with arthritic knee joints with osteotropic ankylosis indicated by X-ray

\subsection{Groups and sample size}


The sample size was estimated to be 99 by a small sample pre-experiment. 18 patients who met the inclusion criteria were randomly divided into 3 groups, 6 subjects in each group to detect the difference of UCSS between Week 8 and the baseline $\quad 1-\beta=1-\operatorname{probF}\left[F_{1-\alpha, G-1, N-G},(G-1), N-G, N V / \sigma^{2}\right]$ value, according to the trial protocol intervention. The mean \pm standard deviation of UCSS reduction was obtained at Week 8 and Week 0 in three groups: $0.33 \pm 0.817,-0.80 \pm 0.837,-1.0$ \pm 1.000 . The common standard deviation is 0.873 , PASS 11 software will be used to estimate sample size by means of the following formula:

Where the significance level $\alpha=0.05$, power of a test $1-\beta=0.80$, group number $G$ =3, $N$ means sample size, $V$ means variance of various horizontal averages, $V=\sum\left(\left(\mu_{i}-\bar{\mu}\right)^{2}\right) / G \quad, \quad \sigma$ is common standard deviation.

$N$ was 99 , taking into account $20 \%$ loss to follow-up, and since the randomised block length was 6 , the sample size should be a multiple of 6 , so the present trial will include 126 patients. The knee joint showing more serious RA symptoms (or the only affected unilateral knee) for each patient will be selected and divided randomly into 3 experimental groups:

(1) Control group (Control with two sham operations): $(n=42)$ These individuals will receive two operations, but $0.9 \%$ saline will be administered instead of PS.

(2) PDT once group: $(n=42)$ Individuals in this group will receive PDT during the first operation, followed by a sham operation similar to that performed for the control group four weeks later.

(3) PDT twice group: $(n=42)$ Individuals in this group will receive PDT twice in 4 weeks.

\subsection{Randomisation and blinding}

After signing the informed consent form, the patients, selected from the rheumatology clinic and ward of our hospital or referred from other hospitals, will enter the screening period. The block randomisation method will be used for 1:1:1 randomisation to ensure a balanced distribution between the groups. The randomisation will be performed by statistical professionals using SAS 9.12 statistical software. The random grouping table will be maintained by a professional nurse at the follow-up hospital centre.

The researchers responsible for evaluations, application of the treatment protocol, and statistical analysis will be blinded to this information. The patients will also be 
blinded to group allocation. All involved (researchers and patients) in the trial will be instructed not to communicate or comment on the group to which the patients will be assigned. Only at the end of the trial will the identities of the groups and the final results be revealed through patient reports. The professional nurse at the follow-up centre will prepare PS or saline according to the random grouping table. The PS solution has the same colour as saline, meeting the double-blind requirements.

If emergency unblinding is deemed to be necessary, for an example, serious adverse events (SAE) like instrument fracture in articular cavity, tissue hematoma, infection, structural damage) occur, the investigators should determine relatedness of an event based on grouping information to the study and actively treat patients.

\subsection{Intervention}

All patients will receive non-steroidal anti-inflammatory drugs, DMARDS or biological agents in accordance with the treatment guidelines.

Patients in the PDT treatment groups will be injected with PS (aminoketoglutarate, $236 \mathrm{mg}, 0.9 \%$ saline dissolved to $5 \mathrm{ml}$ ) in the knee articular cavity before the operation and isometric $0.9 \%$ saline instead of PS in the control group. Two hours later, arthroscopy-guided minimally invasive needle-knife arthroscopy for single-hole exploration and articular cavity lavage with local anaesthesia will be performed, and the synovial tissue will be taken for pathological biopsies before laser irradiation. Laser light-guided fibres will be inserted into the articular cavity via a sleeve. Then, three sites will receive laser irradiation with the knee joint half-curved for a total of 20 minutes: the posterior patella $(10 \mathrm{~min})$ and the medial and lateral condyles of the femurs (5 min each). Laser radiation parameters: wavelength $630 \mathrm{~nm}$, power $150 \mathrm{~mW}$. A second operation will be performed after 4 weeks. More detailed description will be shown in Fig. 2.

\subsection{Evaluation procedure}

The researchers will record postoperative improvement, general condition, including body temperature and respiration, pulse, and heart rates of trial participants through face-to-face follow-ups. The evaluation items for each follow-up are shown in Table 2. All the data will be uploaded to the case report form (CRF) by data collectors who had been rigorously trained. We will also communicate with patients by varies means (eg, wechat, telephone, text message, e-mail) to remind them return to 
hospital for reexamination, which is helpful to reduce the withdrawal rate.

Table 2. Follow-up form

Follow-up time

Baseline

W0

(first operation)

$W 4 \pm 3 d$

(second operation)

$W 8 \pm 3 d$

$W 24 \pm 5 d$

$W 48 \pm 5 d$

\section{Evaluation index}

UCSS, knee joint clinical assessment, DAS28, antibody level, inflammation indicators, X-ray, routine blood test, routine urine test, blood biochemical test, infectious disease screening

Pathological biopsies of synovial tissue

UCSS, knee joint clinical assessment, DAS28, antibody level, inflammation indicators, pathological biopsies of synovial tissue

UCSS, knee joint clinical assessment, DAS28, antibody level, inflammation indicators, blood biochemical test

UCSS, knee joint clinical assessment, DAS28, antibody level, inflammation indicators

UCSS, knee joint clinical assessment, DAS28, antibody level, inflammation indicators, $X$-ray

*W means weekly, d means days

\subsection{Outcome and safety measures}

\subsubsection{Outcome measures}

\section{(1) Primary outcome measure}

The Ultrasound Compound Score of Synovitis (UCSS): According to the EULAR-OMERACT combined scoring system for grading synovitis in rheumatoid arthritis [10]. An improvement in the UCSS compared with the control group will be the primary endpoint.

\section{(2) Secondary outcomes measures}

(1) Knee joint clinical assessment: The circumferential diameter will be measured at 2 $\mathrm{cm}$ on the upper edge of the patella, joint flexion and extension will be measured to assess the range of motion.

(2) DAS28: DAS28-ESR $=0.56 \times$ the total number of tender joints $+0.28 \times$ the total number of swollen joints $+0.70 \times \ln (E S R)+0.014 \times$ general health assessment score

(3) Antibody level: Serum will be collected by centrifugation, and then the RF and anti-CCP antibodies will be detected by a standard market test kit. 
(4) Inflammation indicators: Serum will be obtained by centrifugation, and then ESR and CRP will be detected by standard test kits on the market.

(5)X-ray: X-ray of the knee joint will be used to evaluate bone destruction.

(6) Pathological biopsies of synovial tissue: The synovium of the joint, acquired before laser irradiation in each operation, will be fixed, embedded by dehydration, and sectioned. The synovium will be stained with haematoxylin and eosin for histological evaluation, and synovial histopathology, including synovial cell hyperplasia, inflammatory cell infiltration, and new blood vessels (pannus formation), will be observed.

\subsubsection{Safety measures}

(1) General check-up: Liver function and renal function will be examined to evaluate the effect of basic drug therapy.

(2) Special check-up: After knee joint treatment, wound healing should be observed, and routine blood examination of white blood cells will be performed to assess whether there is infection.

The study will record and handle the PDT-related adverse event or serious adverse event of patients.

\subsection{Data management and statistical analysis}

\subsubsection{Data management}

The project administrator will set out strict procedures to ensure that the data are well preserved and can be traceable. During this trial, the original data from patient and clinical assessments will be collected by data collectors on the CRFs made by the statistician. Furthermore, all of these data will be precisely entered into a password-protected computer 2 weeks after data collection. The data collectors will hand the CRFs and written informed consent forms to administrator for keeping after all of data are entered.

The quality control staff and inspector, as the designated representative of the PI, have the right to monitor whether the trial is carried out in accordance with the trial program. The case report form will be filled out by the researcher, and the data will be recorded in a timely, complete, accurate and clear manner. In accordance with the original data, the inspector shall check the CRF; if there are any errors or omissions, the researcher will be asked to correct them quickly, sign and date the corrections and keep the original record in an easily accessible manner. The CRF form will be 
signed by the inspector, and the first report will be handed over to the clinical trial data manager for data entry and management. For completed case report forms, the researcher and the inspector will transfer the data between administrators into a special record following receipt of the corresponding signatures, and the records will be properly preserved. To ensure confidentiality, data dispersed to all project team members will be blinded of any identifying participant information.

Data entry and modification will be the responsibility of the Department of Health Statistics of Southern Medical University. The data administrator will need to check the CRF table again before the data are entered. Using a query form (DRQ), the researcher should answer any questions as soon as possible and return the DRQ to the clinical inspector, who should properly preserved the document. The data manager shall record two copies of the data and verify that they are identical. The data entry process should be registered and reported in a timely manner, together with the leading researchers, if problems or accidents are found in the input process. The contents of the data range check and logical check will be determined according to the range and interrelation of each index value in the case report table, and the corresponding computer program will be written to ensure that incorrect data cannot be input. If a problem is found, DRQs can be sent out again if necessary. After completing the data entry and verification according to the requirements, the original CRF will be archived and stored in form number order and filed in the search directory for examination. The electronic data file includes the database, the checking program, the analysis program, the analysis result, etc. All original files should be kept in accordance with the Good Clinical Practice (GCP).

\subsubsection{Statistical analysis}

SPSS 20.0 statistical software will be used to analyse the measurement data, described as the means \pm standard deviations $(\bar{x} \pm s)$, and the main evaluation indexes (improvement in the UCSS compared with the baseline value) will be analysed by single-factor variance analysis among the three groups. The ANOVA method will be used for repeated measurement data of the secondary evaluation indicators to explore the principal and interactive effects of grouping and time. Using paired $t$ test at two time points (before and after treatment), multiple time variance points will be analysed by using repeated measurements. Single-factor ANOVA will be used to compare the differences at the same time points after treatment between the three groups. Differences will be statistically significant at $P<0.05$. 


\subsection{Quality Control}

To ensure an integrated, scientific, and effective study, the operators, data collectors, data analysts, and data managers will receive strict and specific training before the implementation of the clinical protocol. The operators who are the essential for ensuring the reliability of the trial, will be required to improve the theory and practical ability of PDT. In addition, to put the subjects at ease during the study, the operators will attempt to prevent any negative reactions on the part of subjects by taking some lessons on communication methods and skills. Data collectors will fill the original data into the CRF in a timely, integrated, and accurate way.

\section{Discussion}

Traditional drugs, including non-steroidal anti-inflammatory drugs, hormones, and disease-modifying anti-rheumatic drugs (DMARDS), have been rapidly developed in the past 10 years and can control the general conditions of most patients with RA; nevertheless, there some patients demonstrate poor curative effects, which greatly increases the difficulty of clinical treatment and reduces the quality of life of patients. Although the refractory joints can be treated by local injection of hormones or arthroscopic synovectomy, these treatments may have substantial shortcomings, and the underlying problems may not be fully resolved. As a new potential therapy for the treatment of synovial hyperplasia in RRA, PDT confers less trauma, stronger targeting, fewer side effects, and more accurate curative effects than hormone injection and synovectomy, and patients tend to tolerate the treatment. PDT has wide application prospect and has already demonstrated promising results, but large deficiencies and fundamental problems remain to be solved.

Now that we have established that refractory synovitis is the key to RRA, early intervention in the long course of RA can be useful for reducing proliferation and migration of FLs and inhibiting inflammatory cell infiltration. The cytotoxic and immunoreactive effects of PDT, which can inhibit synovial hyperplasia and pannus formation and thus reducing cartilage and bone destruction, are critical for the treatment of RRA. In clinical practice, the author found that the light dose and the time and scope of PDT are not easy to control, that long periods of clinical operation and experience are required to judge the outcomes, and that the therapy cannot completely eliminate synovial hyperplasia.

To date, almost all studies of PDT for RA consist of basic research and arthritis 
animal model experiments, which have confirmed the effect of PDT on arthritis, demonstrating a lack of research on clinical efficacy evaluation. In view of this, the author envisages that this study will clarify the short- and long-term clinical efficacy of PDT in the treatment of RA synovitis, explore the treatment frequency and treatment course, and finally form a code of practice to provide scientific data on a new and feasible therapy for the treatment of RRA synovial hyperplasia.

We believe that with an increasing amount of basic research and clinical trials, the long-term efficacy and technical problems of PDT in the treatment of RRA synovial hyperplasia will be addressed and that PTD will be widely used in the clinic.

\section{Strengths and limitations of this study}

(1) The main aim of the trial is to evaluate the short-, medium- and long-term clinical efficacy of photodynamic therapy (PDT) in the treatment of refractory RA (RRA) synovial hyperplasia and synovitis for about one year of follow-up.

(2) An improvement in the The Ultrasound Compound Score of Synovitis (UCSS) compared with the baseline will be the primary endpoint based on the EULAR-OMERACT combined scoring system for grading synovitis in RRA.

(3) PDT has the advantages of minimal trauma, strong targeting, few side effects, and definite curative effects, making it more acceptable to patients compared with steroid injections or arthroscopic synovectomy.

(4) Participants from more than eight provinces in China will be included.

(5) It's only a single-centre trial.

\section{Abbreviations}

RRA: Refractory Rheumatoid Arthritis; PDT: photodynamic therapy; UCSS: The Ultrasound Compound Score of Synovitis; DAS28: Disease Activity Score in 28 Joints; EULAR-OMERACT: European League Against Rheumatisms-Outcomes Measures in Rheumatology; PS: photosensitizer; Ros: Reactive oxygen species; ARA: American Rheumatism Association; DMARDs: Disease-modifying antirheumatic drugs; SAE: Serious Adverse Events; CRF: Case Report Form; ESR: Erythrocyte Sedimentation Rate; CCP: Cyclic Citrullinated Peptide; CRP: C-reactive protein; DRQ: Query Form; GCP: the Good Clinical Practice; ANOVA: Analysis of Variance; CRDC: Chinese Rheumatism Date center. 


\section{Declarations}

\section{Ethics approval and consent to participate}

The protocol was approved by the Medical Ethics Committee of Integrated Hospital of Traditional Chinese Medicine, Southern Medical University. An electronically written consent was obtained from all participants before they participating in the study.

\section{Consent for publication}

Not applicable

\section{Author contributions}

CX: Principle investigator, Writing-review \& editing, Project implementation and administration.

XZ: PI assistant, Writing-original draft, Data curation, Quality control.

EC: Research Doctor, Writing-original draft, Minimally invasive needle scalpel operator.

FZ and YY: Research Doctor, Minimally invasive needle scalpel operator.

YC and YB: Research Doctor, Joint ultrasound and report.

KL: Laboratory technician, Antibody test.

YX and LW: Surgical nurse, Equipment manager.

LL: PDT Implementation.

XQZ: Imaging Examination and report.

FY: Follow-up Centre Professional Nurse, Drug preparation.

JZ: Data Statistics.

KY: Referring patients, Telephone follow-up.

All the authors discussed, revised and approved the final manuscript.

\section{Patient and public involvement}

Patients included in the trial study will not be involved in developing plans for recruitment, design, reporting, implementation, dissemination.

\section{Project Funding}

Supported by the Training Program of the Clinical Research Plan of Southern Medical University (No. LC2016PY053) belongs to high-level university funding for scientific research, which is from the Finance Special Fund of Guangdong Provincial Government. The funding source had no role in the design of this study and will not have any role during its execution, analyses, interpretation of the data, or decision to submit results. 


\section{Protocol version}

Issue date: Sep 1st, 2020 Protocol amendment number: V1.6

\section{Competing interests}

All authors declare they don't have any other financial and personal conflicts of interest with this manuscript.

\section{Availability of data and material}

The principal investigator, Changhong Xiao, Professor, PhD, will have access to the final trial dataset. And the raw data will be shared in one year after the end of the trial, including the Clinical Trial Public Management Platform ResMan (www.medresman.org) or Chinese Rheumatism Date center (CRDC).

\section{Trial Status section}

Participant recruitment and Follow-up is still in progress.

\section{Acknowledgments}

Not applicable.

\section{References}

[1] Tommy Tsang Cheung, lain B. Mclnnes, Future therapeutic targets in rheumatoid arthritis? Semin Immunopathol, 2017, 39(4):487-500.

[2] Lundquist LM, Cole SW, Augustine JM. Critical appraisal of efficacy and safety of abatacept in the treatment of refractory rheumatoid arthritis[J]. Open Access Rheumatol, 2012,4:9-19.

[3] Hajialilo M, Ghorbanihaghjo A, Valaee L, et al. A double-blind randomized comparative study of triamcinolone hexacetonide and dexamethasone intra-articular injection for the treatment of knee joint arthritis in rheumatoid arthritis[J]. Clin Rheumatol, 2016,35(12):2887-2891.

[4] Shankar V, Sharma P, Mittal R, et al. Effectiveness of arthroscopic elbow synovectomy in rheumatoid arthritis patients: Long-term follow-up of clinical and functional outcomes[J]. J Clin Orthop Trauma, 2016,7(Suppl2):230-235.

[5] Xingshu Li, Songyi Lee, Juyoung Yoon.Supramolecular photosensitizers rejuvenate 
photodynamic therapy. Chem. Soc. Rev., 2018,47: 1174.

[6] Choudhary S, Nouri K, Elsaie ML. Photodynamic theragy in dermatology: a review[J]. Lasers Med Sci, 2009,24(6):971-980.

[7] Arnett FC , Edworthy SM , Bloch DA , et al. The American Rheumatism Association 1987 revised criteria for the classification of rheumatoid arthritis. Arthritis Rheum 1988,31 (3): 315-24.

[8] Smolen JS, Aletaha D, Koeller M, et al. New therapies for treatment of rheumatoid arthritis[J]. Lancet, 2007,370:1861-1874.

[9] Farah Z, Ali S, Price-Kuehne F, et al. Tocilizumab in refractory rheumatoid arthritis: long-term efficacy, safety, and tolerability beyond 2 years[J]. Biologics, 2016,22(10)59-66.

[10] D'Agostino MA,et al. Scoring ultrasound synovitis in rheumatoid arthritis: a EULAR--OMERACT ultrasound taskforce-Part 1: definition and development of a standardised, consensus-based scoring system. RMD Open. 2017,3(1):e000428. 


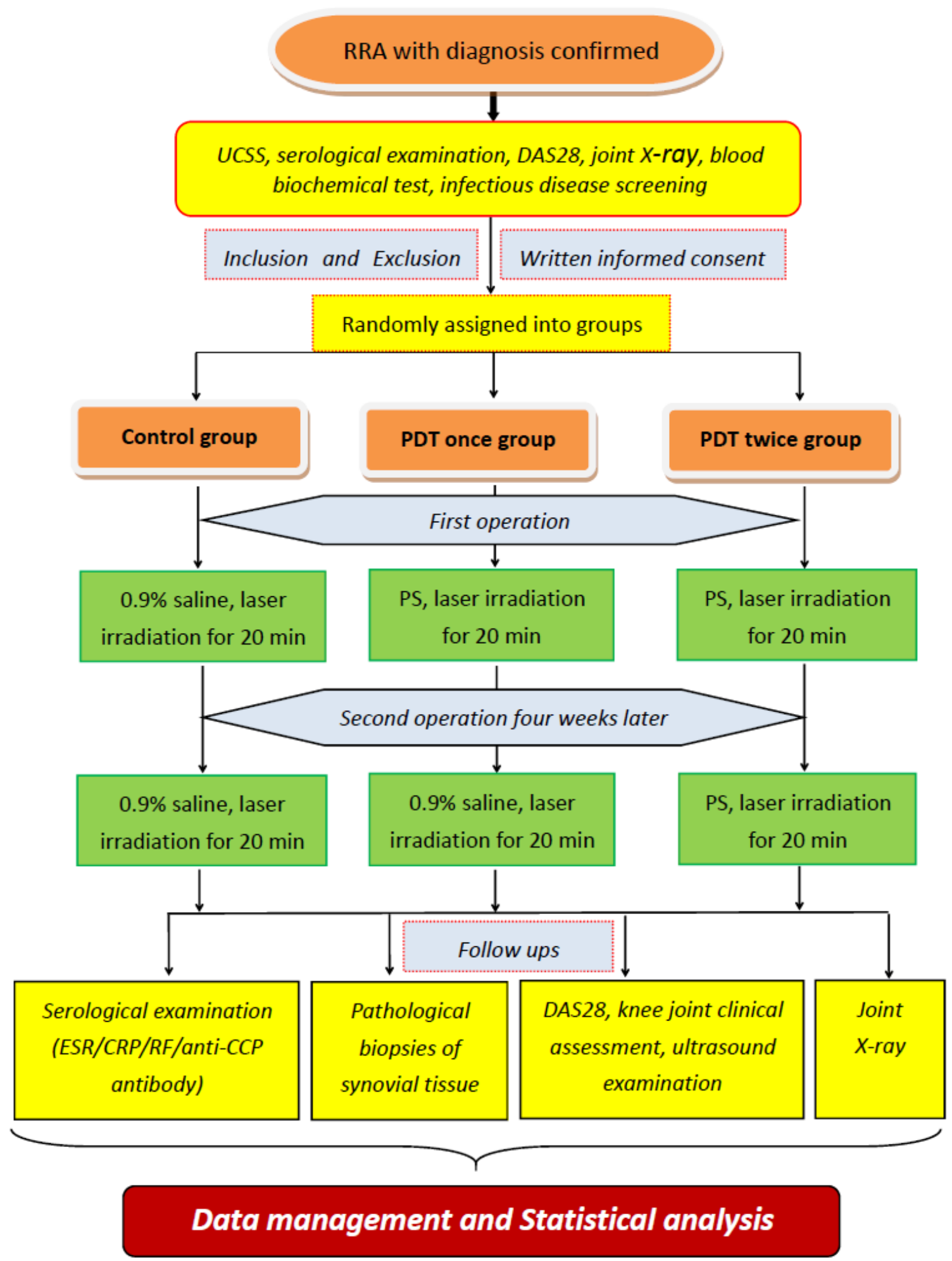

\section{Figure 1}

Trial flow diagram. 


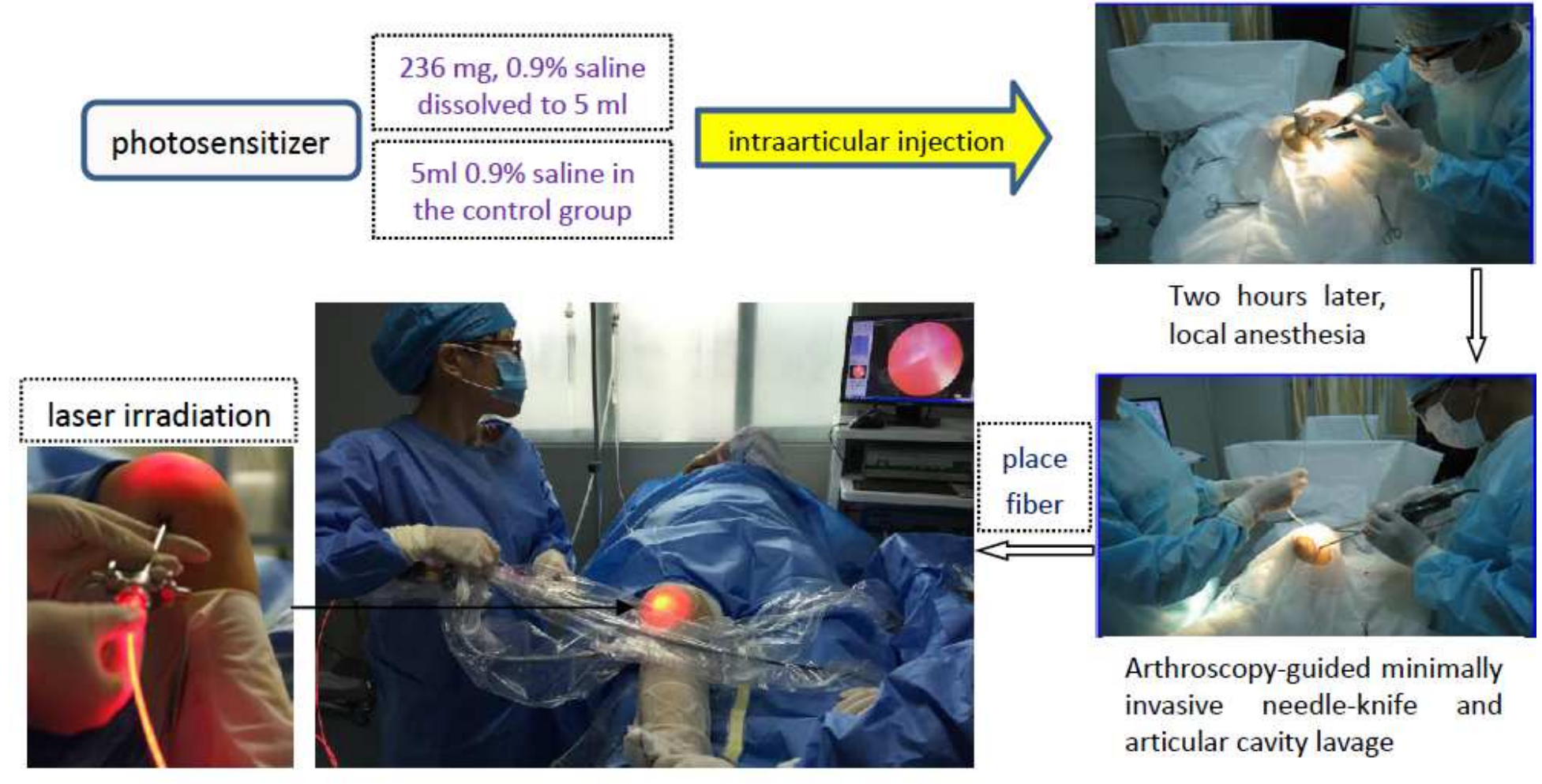

Figure 2

Photodynamic therapy process and scenarios. 